\title{
Aplicación del marco de referencia FMPEIR para la determinación del estado ambiental de El Salvador
}

\author{
José Ricardo Calles \\ Recibido: 15/08/2013 - Aceptado: 10/09/2013
}

\section{Resumen}

El estudio sobre el estado ambiental de El Salvador requiere de un instrumento analítico que establezca un enlace lógico entre lo social y económico, como factores que ejercen presión sobre los recursos naturales, y que deben considerarse como las causas de los problemas ambientales, y que a su vez determinan las perspectivas de sostenibilidad nacional. Un instrumento analítico lo constituye el marco de referencia conocido como Fuerza Motriz-Presión-Estado-Impacto-Respuesta (FMPEIR), el cual ha sido desarrollado por el Programa de las Naciones Unidas para el Medio Ambiente (PNUMA) en sus evaluaciones ambientales integrales.

Lo que se presenta en este artículo es la aplicación del marco de referencia FMPEIR para ordenar y agrupar, de manera lógica, los factores que actúan sobre el ambiente, los efectos producidos por las acciones humanas en los ecosistemas y en los bienes ambientales, así como el impacto que esto genera en la salud humana y en la propia naturaleza, además de las intervenciones por parte de la sociedad y del Gobierno para enfrentar los problemas generados por las acciones humanas. La aplicación del instrumento FMPEIR ha permitido analizar las cuestiones ambientales a través de un enfoque sistémico y un marco conceptual enfocado en las tensiones ambientales inherentes a las dinámicas del desarrollo humano, permitiendo construir un panorama general del estado situacional del ambiente salvadoreño y revelar sus tendencias, las dimensiones humanas que generan

\section{Abstract}

The study on the environmental status of El Salvador requires an analytical tool that establishes a logical link between social and economic factors that put pressure on natural resources which are to be considered as the "causes" of environmental problems. These in turn determine national sustainability prospects. The framework known as Driving Force-Pressure-State-Impact-Response (DPSIR) is an analytical instrument that has been developed by the United Nations Program for Environment (UNEP) to be applied in integrated environmental assessments.

This paper is the application of the DPSIR framework for logically sorting and grouping the factors that affect the environment, the effects of human actions on ecosystems and environmental assets and the impact they have on human health and nature. It also takes into account interventions by society and government to address the problems caused by human actions.

The application of DPSIR instrument has allowed analyzing environmental issues through a systematic approach and a conceptual framework focused on the tensions of environmental dynamics inherent in human development. It allows to build a situational overview of the state of the environment and to reveal Salvadoran trends as well as the human dimensions that generate their change and future prospects to protect and improve their condition. 
su cambio y las perspectivas de futuro para proteger y mejorar su condición.

\section{Palabras clave}

Gestión Ambiental, política ambiental, protección del medio ambiente, conservación de los recursos naturales, control ambiental, áreas protegidas.

\section{Keywords}

Environmental management, environmental policy, environmental preservation, conservation of natural resources, environmental control, protected areas.

\section{Introducción}

En el año 2012, la Universidad Tecnológica de El Salvador realizó la investigación denominada "Libro Verde Utec 2012 Estado del medio ambiente y perspectivas de sostenibilidad" con el propósito de proveer información que facilite el análisis y la reflexión acerca del estado actual y las perspectivas del ambiente en El Salvador, y de esa forma proponer posibles soluciones a los problemas existentes, así como contribuir al monitoreo continuo del estado ambiental.

El enfoque metodológico de esa investigación ha sido una adaptación del método que propone el Programa de las Naciones Unidas para el Medio Ambiente (PNUMA) para los informes de Perspectivas del medio ambiente (GEO, por sus siglas en inglés), sustentado en un proceso interdisciplinario que combina, interpreta y comunica conocimiento de diferentes disciplinas científicas para comprender los vínculos entre el estado del ambiente y el bienestar humano (PNUMA, 2007). Ese método se basa en el marco de referencia FMPEIR (Fuerza Motriz- Presión-Estado-Impacto-Respuesta), que permite ordenar y agrupar, de manera lógica, los factores que actúan sobre el ambiente, los efectos producidos por las acciones humanas en los ecosistemas y en los bienes ambientales, así como el impacto que esto genera en la salud humana y en la propia naturaleza, además de las intervenciones por parte de la sociedad y del Gobierno para enfrentar los problemas generados por las acciones humanas.

En los años $2002^{1}$ y $2006^{2}$, la Oficina Regional para América Latina y el Caribe del PNUMA (ORPALC) ha proporcionado asistencia técnica y financiera al Gobierno de El Salvador para apoyar el desarrollo de evaluaciones GEO nacionales que aplican este marco de referencia analítico. Dado que desde el 2006 no existe evidencia de otro informe nacional, la Utec ha intentado, mediante la aplicación del instrumento analítico FMPEIR, responder a la necesidad de brindar información para apoyar la gobernanza ambiental y la transversalidad de las preocupaciones ambientales en los sectores social y económico, y para apoyar las metas de desarrollo convenidas internacionalmente, facilitando la interacción entre la ciencia y la política pública a partir de una visión que asegure la disponibilidad de recursos para las futuras generaciones.

\section{El marco de referencia FMPEIR}

El proceso metodológico de aplicación del marco de referencia FMPEIR se ilustra en la figura 1. El modelo pretende reflejar los componentes clave de la cadena causa y efecto, compleja y multidimensional, espacial y temporal, que caracterizan a las interacciones entre la sociedad y el ambiente (PNUMA, 2007). Además, trata de mejorar la comprensión de la sociedad sobre los vínculos entre el ambiente y el desarrollo, el bienestar humano y la vulnerabilidad a los cambios ambientales. La metodología requiere la compresión precisa de los aspectos incluidos en el marco de referencia que darán respuesta a cinco preguntas básicas: (i) ¿Qué le está pasando al ambiente? (ii) ¿Por qué está ocurriendo? (iii) ¿Cuáles son las

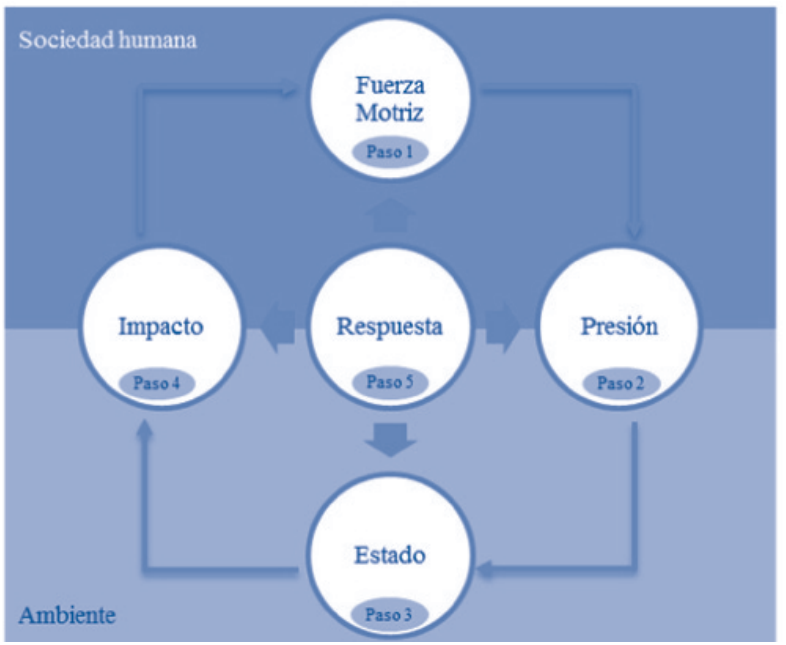

Figura 1. Marco de referencia FMPEIR Fuente: Adaptado de Pintér, et al., 2007. 
consecuencias para el ambiente y la humanidad? (iv) ¿Qué se está haciendo? y (v) ¿Qué medidas podrían tomarse para asegurar un futuro más sostenible? La respuesta a estas preguntas permite construir un panorama general del estado situacional del sistema ambiental salvadoreño y revelar sus tendencias, las dimensiones humanas que generan cambio en ellos, las perspectivas de futuro y las opciones de política para proteger y mejorar su condición. Por esta razón se deben definir cada uno de ellos, según lo establece PNUMA-Orlac (2008) (Tabla 1).

Tabla 1. Definiciones de los aspectos FMPEIR

\begin{abstract}
A veces referida como fuerza indirecta o subyacente. Está relacionada con procesos fundamentales de la Fuerza sociedad, que promueven actividades que tienen un impacto indirecto sobre el medio ambiente. Fuerzas clave motriz incluyen: demografía de la población; conductas de consumo y producción; innovación científica y tecnológica; demanda económica; mercado y comercio; patrones de distribución; marcos institucionales y sociopolíticos; y sistemas de valores.

Se refiere a las fuerzas económicas y sociales subyacentes, tales como el crecimiento de la población, el consumo o la pobreza. Desde el punto de vista político, la presión constituye el punto de partida para enfrentar

Presión los problemas ambientales. La información sobre la presión tiende a estar más disponible, puesto que proviene de bases de datos socioeconómicos. El conocimiento de los factores de presión busca responder a la pregunta: ¿Por qué está sucediendo esto?

Estado

Se refiere a la condición del medio ambiente como resultado de la presión; por ejemplo, el nivel de contaminación atmosférica, la erosión del suelo o la deforestación. La información sobre el estado del medio ambiente responde a la pregunta: ¿Qué le está sucediendo al medio ambiente?

Es el efecto producido por el estado del medio ambiente en aspectos como la calidad de vida y la salud humana, Impacto el mismo medio ambiente, el ambiente construido y la economía urbana local. Por ejemplo, el aumento en la erosión del suelo tendrá una o más consecuencias: disminución en la producción de alimento, aumento en su importación, incremento en el empleo de fertilizantes y desnutrición. La información sobre el impacto responde a la pregunta: ¿Cuáles son las consecuencias para el ambiente y la humanidad?

Corresponde a las acciones colectivas o individuales que atenúan o evitan impactos ambientales negativos; corrigen el daño causado al medio ambiente; conservan los recursos naturales o contribuyen a mejorar la calidad Respuesta de vida de la población local. Las respuestas pueden incluir actividades en la reglamentación, costos ambientales o de investigación; opinión pública y preferencias del consumidor; cambios en las estrategias administrativas y el suministro de información relacionada con el medio ambiente. Los instrumentos incluidos en esta categoría intentan responder a la pregunta: ¿Qué se está haciendo?
\end{abstract}

\section{Resultados de la aplicación del marco de referencia FMPEIR}

Para la aplicación del marco de referencia FMPEIR desarrollada en la investigación "Libro Verde Utec 2012: Estado del medio ambiente y perspectivas de sostenibilidad", se realizó una revisión bibliográfica en la que se exploró un conjunto de fuentes de información secundaria, consistente en bases de datos y cifras derivadas de estudios e informes relevantes para los propósitos de la investigación, los cuales se detallan a continuación: (i) bases de datos estadísticos de la FAO; (ii) datos básicos de la Agencia Europea de Medio Ambiente (AEMA); (iii) selección de datos ambientales de la OCDE; (iv) indicadores básicos GEO; (v) indicadores temáticos de la UN CSD.

El procedimiento para la recolección de información estuvo basado en el método Go-CART, el cual permite planear la búsqueda de datos secundarios externos y responde a las iniciales de las siguientes palabras inglesas 
(Moreno-Manzanaro, 2005): (i) Goals-metas: definir temas y conceptos oportunos para la investigación; (ii) Objectivesobjetivos: reunir toda la información disponible y clasificarla en apartados concretos; (iii) Characteristics-características: determinar las características de la información que se precisa; (iv) Activities-actividades: realizar un esquema de las personas y lugares que se deben visitar; (v) Reliabilityconfiabilidad: consultar varias fuentes para cerciorarse de que se trata de información útil; y (vi) Tabulation-tabulación: documentar todas las fuentes de datos.
A continuación se exhiben los resultados de la aplicación del marco de referencia FMPEIR.

\section{Fuerzas motrices}

Las fuerzas motrices son de carácter social (demografía y educación) y económico (pobreza, empleo, salarios, acceso al agua potable, disponibilidad y acceso económico a los alimentos), y para cada una de ellas se han considerado los siguientes aspectos:

\section{Demografía}

Evolución de la población

Estructuras de edades de la población
Hacia el año 1950, la población censada que se registró en El Salvador fue de 1.855.917 habitantes (63\% población rural), número que alcanza los 5.774 .113 en el censo de 2007 (62,7\% área urbana y $37,3 \%$ rural) (UNFPA, 2010).

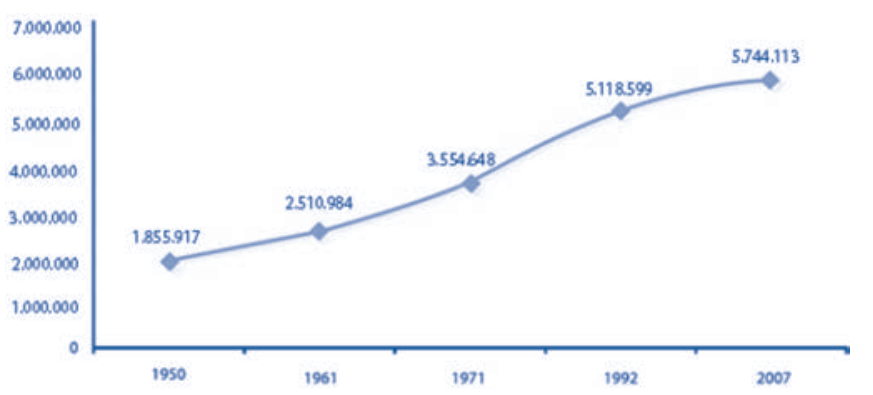

Figura 2. Población total según censos de 1950, 1961, 1971, 1992 y 2007

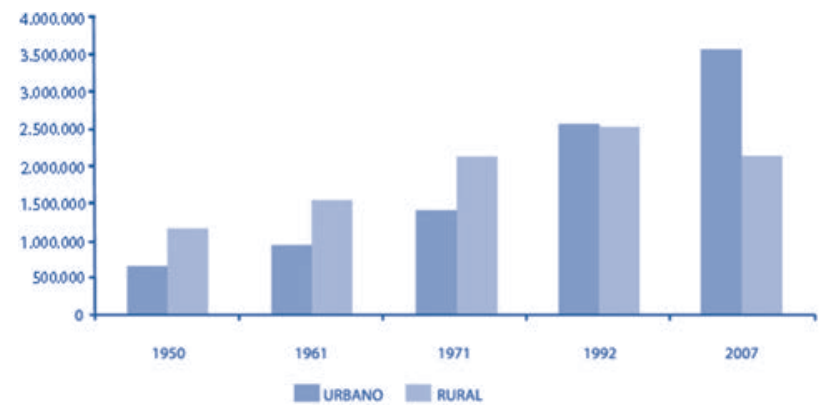

Figura 3. Población total por área de residencia geográfica, según censos de 1950, 1961, 1971, 1992 y 2007

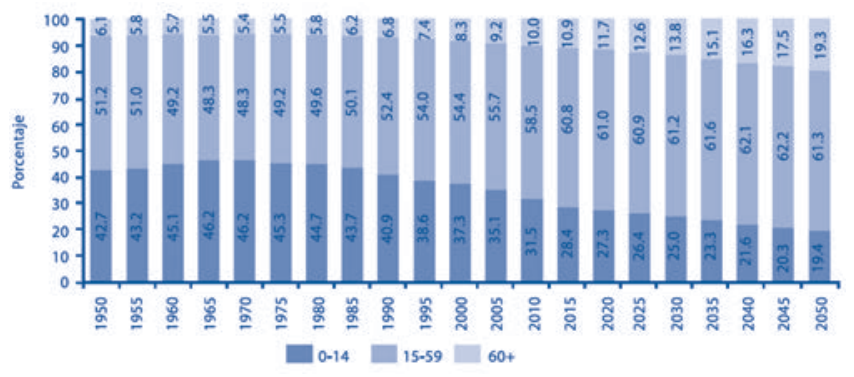

Figura 4. Distribución porcentual de la población por grupos etarios, 1950-2050 


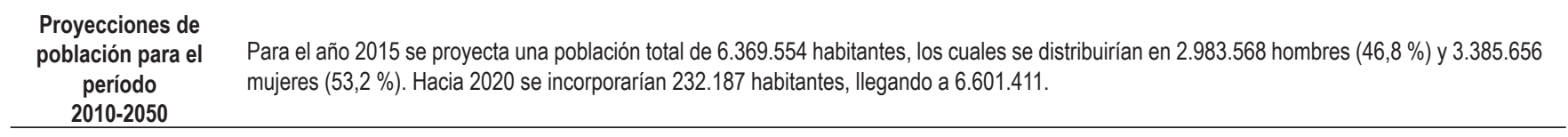

Fuente: UNFPA, 2010

\section{Educación}

En El Salvador existen 652.928 personas que no saben leer ni escribir, lo que representa una tasa de analfabetismo de aproximadamente 12,8\%. En el área urbana es de $8,2 \%$ y en lo rural de $20,7 \%$.

\section{Asistencia escolar}

Escolaridad promedio
La escolaridad promedio a escala nacional es de 6.2

La tasa para el 2011 fue de 32,4 \% del total de población de 4 años y más, lo que representa un total de 1.884 .913 alumnos que asistieron a un centro educativo formal en todo el territorio nacional. La tasa de asistencia escolar específica de los hombres a escala nacional es de 34,9 $\%$ y de las mujeres de $30,1 \%$. grados. En el AMSS es de 8.3 grados, 7.3 grados para el área urbana y 4.4 para el área rural.

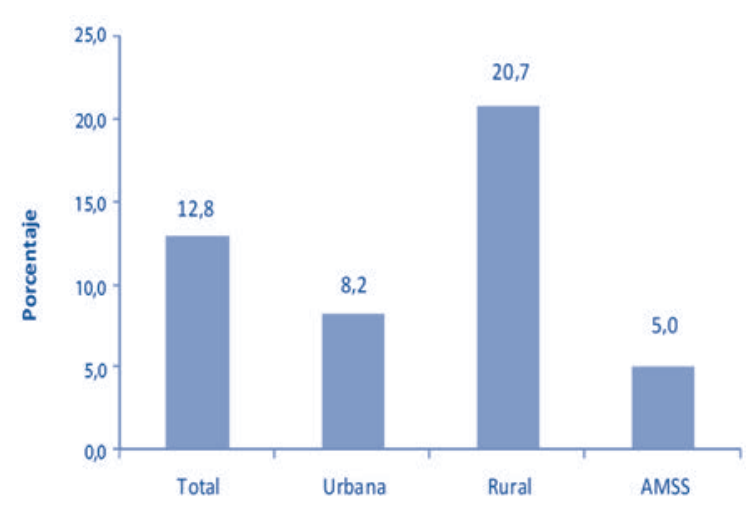

Figura 5. Población Analfabeta por área

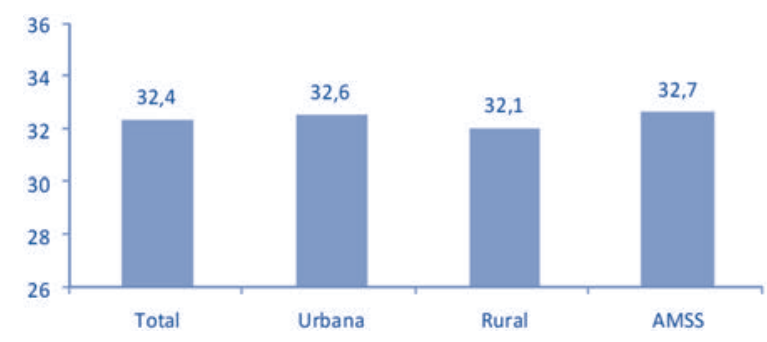

Figura 6. Tasa de asistencia escolar por área (\%)

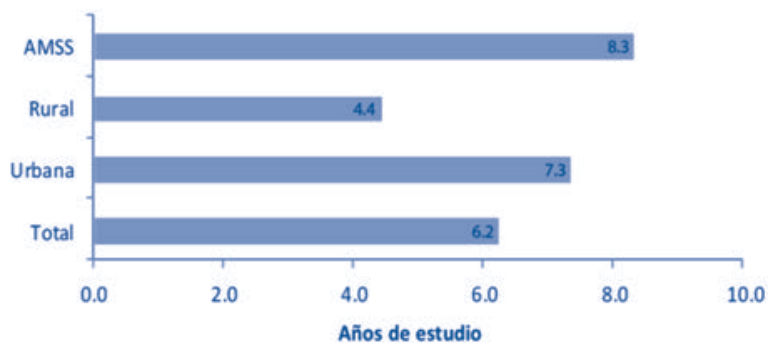

Figura 7. Escolaridad promedio por área

Fuente: Digestyc, 2012. 
Una vez detalladas las fuerzas motrices de carácter social, se exhiben a continuación las fuerzas motrices de naturaleza económica, como son: la pobreza, el empleo, los salarios, acceso al agua potable, disponibilidad y acceso económico a los alimentos.
Pobreza

Empleo

Salarios
Pese a que las tasas de pobreza ahora son bastante menores que en 1991, en 2009 todavía existían en el país más de 2.6 millones de personas pobres, con ingresos inferiores al costo de la canasta básica de consumo. Los niveles de pobreza son mayores en el área rural, donde el $53 \%$ de la población se encuentra en esa condición (figura 8).

La tasa de desempleo en 2009 fue de $7,3 \%$, relativamente baja con respecto a naciones con desarrollo humano alto. Sin embargo, la tasa de subempleo fue de $47 \%$. La incidencia del subempleo, como muestra el gráfico 8 , es distinta para diferentes grupos poblacionales. La incidencia del subempleo es mayor para los jóvenes entre 15 y 24 años de edad, la población de mujeres y la que reside fuera del AMSS.

A escala nacional, se evidencian fuertes asimetrías en los salarios en diversos sectores. El gráfico 9 compara los salarios promedio registrados en la agricultura, la construcción, la industria manufacturera y el comercio. El menor de todos corresponde al salario agrícola. No es casual, por lo tanto, que los mayores niveles de pobreza en el país correspondan al segmento de trabajadores asalariados agrícolas. Según la EHPM de 2009 (Minec y Digestyc, 2010), el $58 \%$ de las personas que trabajan en dicho sector se encuentran en pobreza, frente al $34 \%$ en el sector construcción; $31 \%$ en la industria manufacturera y $26 \%$ en el sector de comercio, hoteles y restaurantes.

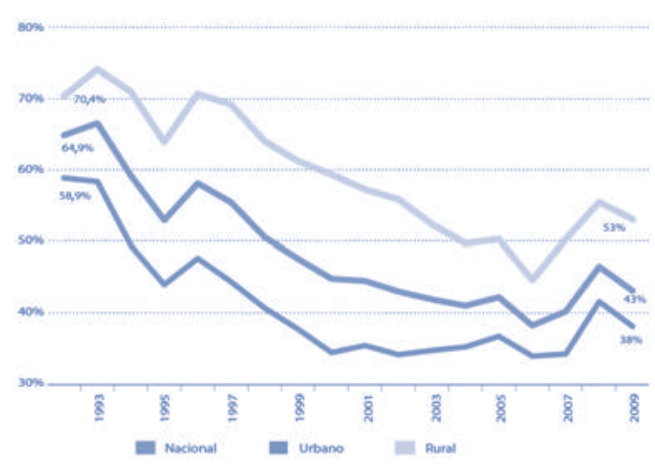

Figura 8. \% de personas en pobreza, por áreas de residencia

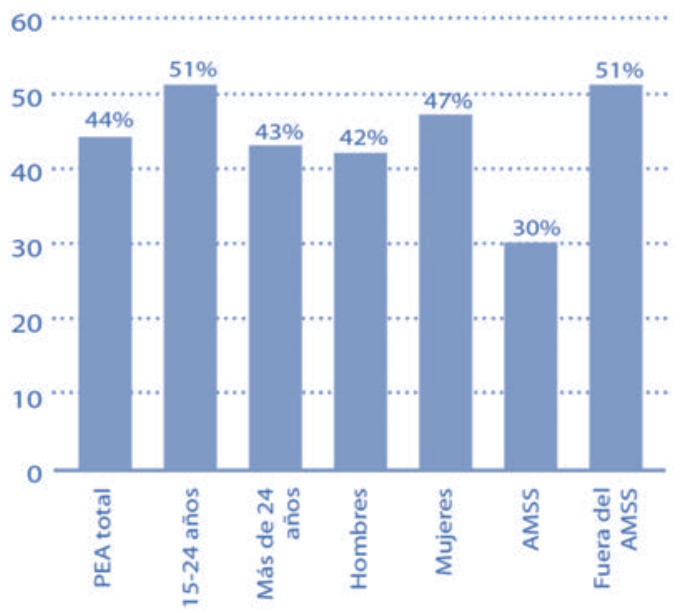

Figura 9. Proporción de la población con subempleo, 2009

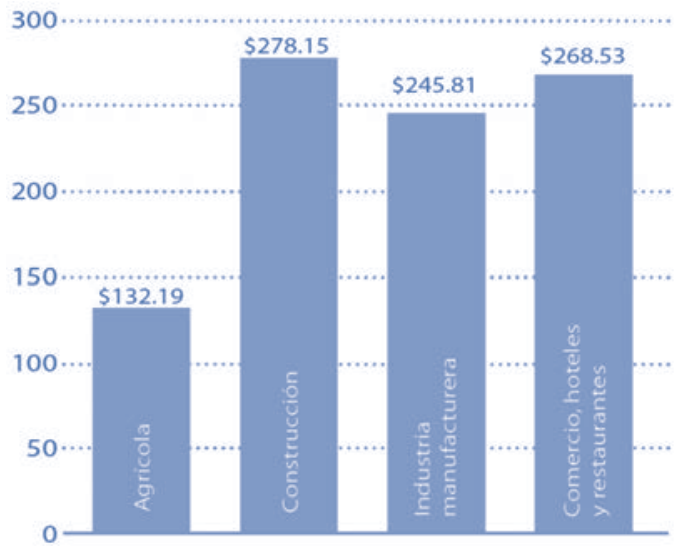

Figura 10. Salarios promedio 


\section{Acceso a agua potable}

\section{Disponibilidad de alimentos}

\section{Acceso económico} a los alimentos
Los hogares que cuentan con el acceso al servicio de agua por cañería a escala nacional son el 83,8 $\%$ (gráfico 10). Los que se abastecen con agua de pozo representan el 8,9\%, mientas que los que lo hacen a través de otros medios el 7,3\% de los hogares. El 93,1\% de los hogares del área urbana cuentan con acceso al agua por cañería. En contraste, en el área rural solo el $66,4 \%$ de los hogares cuenta con acceso al servicio de agua por cañería, mientras que el $17,4 \%$ se abastece con agua de pozo y el $16,2 \%$ lo hace por otros medios.

Depende de la agricultura y, cada vez más, de las importaciones. Esto se debe a que en las últimas cuatro décadas la agricultura de El Salvador ha tenido la tasa de crecimiento más baja en Centroamérica: entre 1961 y 2009, la producción agrícola aumentó $1,6 \%$ en promedio al año. La participación del sector agrícola en el PIB se redujo, de $17 \%$ en 1990, a $13 \%$ en 2009 .

El gráfico 11 muestra la evolución de la producción y el consumo aparente per cápita de granos básicos y carne para el período 1980-2007. El crecimiento del consumo aparente fue mayor que el de la producción interna, por lo que se observa un crecimiento en el déficit de producción. Este déficit ha sido compensado con el crecimiento de las importaciones de alimentos.

Durante el período 2007-2009, el acceso económico a los alimentos se vio deteriorado por diversos factores relacionados con la crisis alimentaria y la crisis económica mundial, que tuvieron importantes efectos en los niveles de pobreza de la población. Así, el precio de los alimentos en mercados internacionales tuvo un fuerte incremento entre enero de 2007 y julio de 2008. El gráfico 12 muestra la evolución de precios de alimentos en El Salvador, que refleja el fuerte incremento en los precios internacionales. El incremento fue de 19 $\%$ durante el período de enero de 2007 a julio de 2008, manteniéndose en 2009 precios superiores a los de 2007.

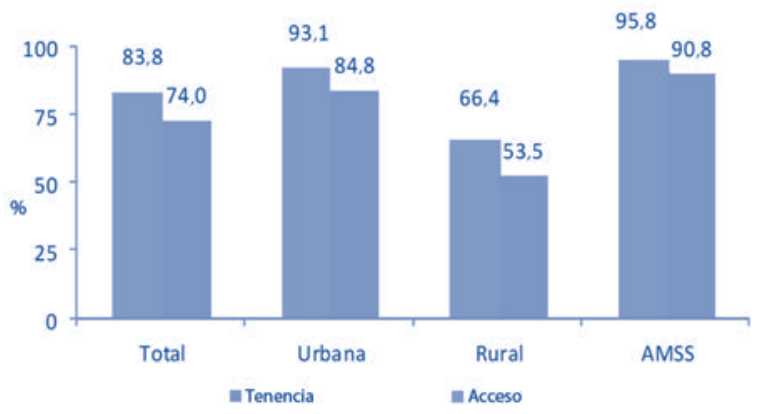

Figura 11. \% de hogares que dispone de agua por cañería (área)

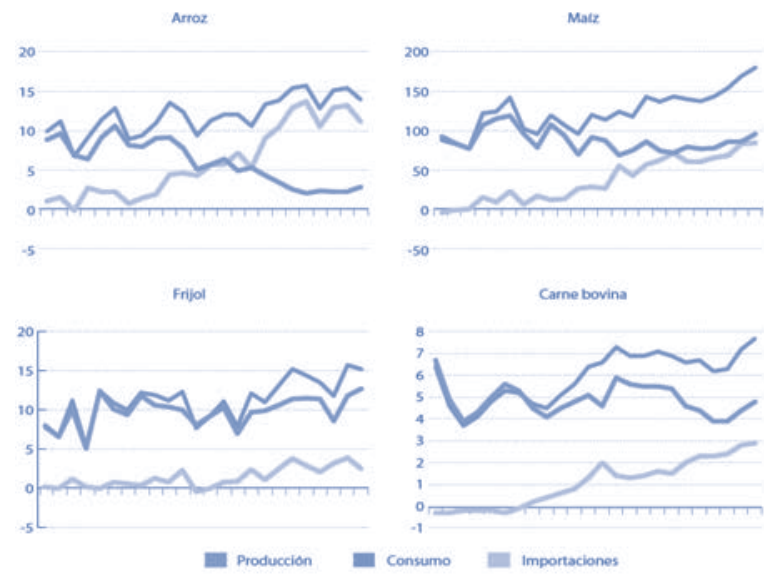

Figura 12. Producción, consumo e importaciones de granos básicos y carne 1980-2007 (kg/persona)

300

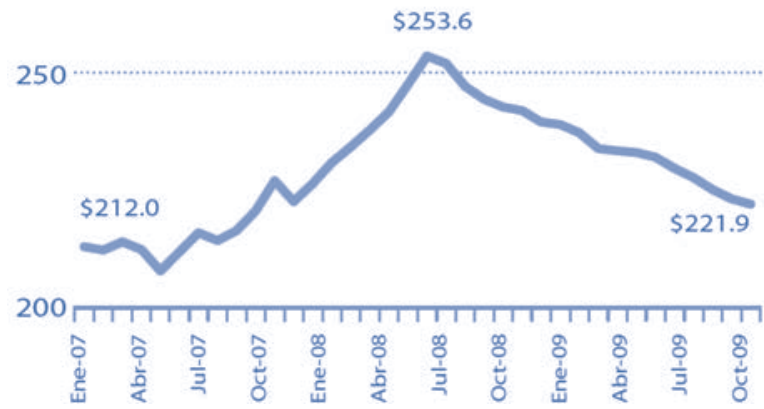

Figura 13. Evolución del IPC de alimentos

(En US\$, para el periodo de enero de 2007 a diciembre de 2009)

Fuente: Sistema de las Naciones Unidas, 2011. 


\section{Presiones - ¿Por qué está ocurriendo la degradación ambiental?}

De acuerdo con lo establecido anteriormente, las condicionantes sociales y económicas constituyen las fuerzas motrices que generan las presiones sobre el ambiente; es decir, las características demográficas y educativas, así como la pobreza, el empleo, los salarios, la disponibilidad y el acceso económico a los alimentos, se encuentran ejerciendo una influencia directa en las condiciones del medio ambiente. A partir de esto, las presiones derivadas de estos aspectos socioeconómicos (figura 14).

Urbanización. Un efecto directo del aumento demográfico es el acelerado proceso de urbanización del país. Mientras que en 1950 solo una de cada tres personas $(36,4 \%)$ residía en un centro urbano, en 2007 esta proporción se elevó a casi dos de cada tres (62,7\%). Para 2030 se estima que la relación se habrá invertido totalmente, de manera que solo una de cada cuatro residirá en asentamientos rurales.
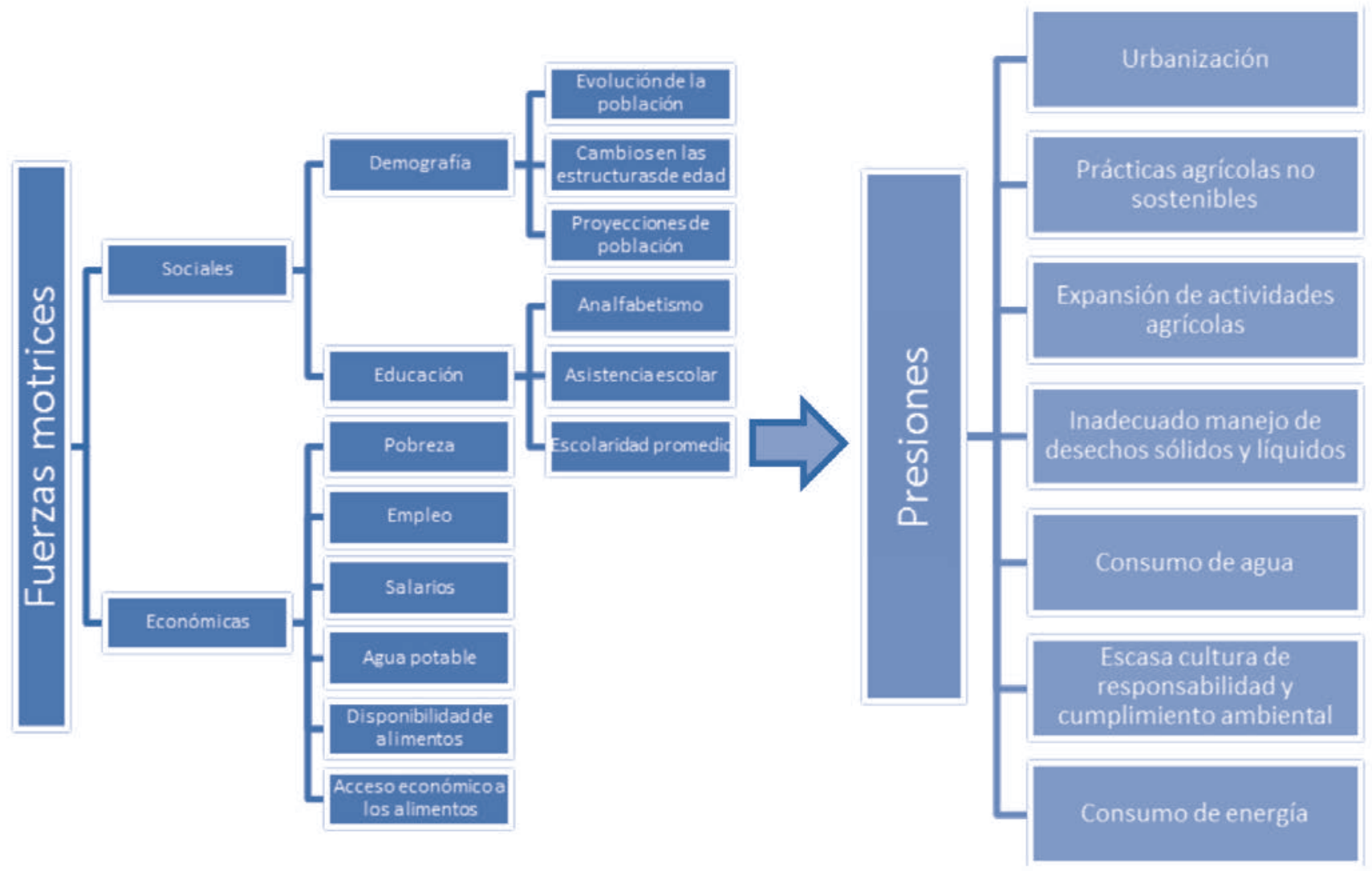

Figura 14. Fuerzas motrices y presiones

Prácticas agrícolas no sostenibles. Los incendios forestales y las quemas agrícolas son una problemática recurrente, que afecta a los ya escasos recursos forestales del país, al provocar la destrucción y graves daños a los bosques naturales y seminaturales, plantaciones forestales y las áreas naturales protegidas. Según la Comisión Nacional para los Incendios Forestales y el MARN, durante el período 2001-2012 los incendios ocurridos afectaron
38.965 hectáreas, siendo el año 2006 el que registró mayor incremento de ocurrencia con 117 incendios, que afectaron un total de 8.473 hectáreas.

Expansión de actividades agrícolas. La caña de azúcar es uno de los cultivos más extensos en el país que ha experimentado un crecimiento acelerado desde el año 2000, teniendo implicaciones sobre los recursos forestales, específicamente porque, para su 
establecimiento, ha eliminado áreas boscosas y de regeneración natural. En términos generales, se han identificado algunas de las principales causas subyacentes a la deforestación y la degradación, y son: (1) migración y remesas que distorsionan los precios de las tierras; (2) pobreza y falta de oportunidades económicas en áreas cercanas a los bosques; (3) información cartográfica confusa e inaccesible (tenencia de la tierra) sobre los límites de los bosques.

Inadecuado manejo de desechos sólidos y líquidos. Con la prohibición de los botaderos a cielo abierto, el volumen de desechos depositados a cielo abierto disminuyó a la mitad: de 1.611 toneladas de desechos diarias en 2007 a unas 800 toneladas en 2011; una cantidad todavía alta. El vertido de aguas residuales $\sin$ tratar deteriora las aguas superficiales, encarece su potabilización, limita su uso en la producción y representa un serio riesgo para la salud. Los altos niveles de coliformes fecales en aguas superficiales es un indicador de contaminación por aguas negras y se encuentran asociados a enfermedades gastrointestinales, que son una de las primeras diez causas de muerte en el país y la segunda causa de enfermedad. Aunque las descargas industriales representan apenas el $2 \%$ de los vertidos, contienen en algunos casos componentes físicos, biológicos y químicos, incluyendo metales pesados, que dificultan su tratamiento.

Consumo de agua. El agua que se extrae del territorio se orienta principalmente para las actividades agropecuarias, alcanzando la demanda el $46 \%$ del total del recurso hídrico que se extrae; para consumo de los hogares se orienta el 34 $\%$, y la industria requiere el $20 \%$ (FAO, 2000). Es importante señalar que el país no cuenta con información actualizada y coherente de la demanda (uso) del agua. De acuerdo con los datos de Anda (2011), en los últimos diez años, el sector residencial es el que reporta mayor consumo, con un promedio de $82 \%$ del agua potable que se produce y distribuye; seguido de las explotaciones privadas, con un $9 \%$. El total del agua que produjo Anda en 2011 alcanzó los 226.6 millones de metros cúbicos.

Escasa cultura de responsabilidad y cumplimiento ambiental. Esta situación es reconocida en el ámbito poblacional, como lo muestra parte de los resultados de la Primera Encuesta Nacional sobre Conocimientos, Comportamientos, Percepciones y Prácticas de la población salvadoreña sobre Medio Ambiente y Riesgos (MARN, 2011b), en donde, ante la pregunta referida a cuáles son las causas del deterioro del medio ambiente, la "falta de información de la gente y poca educación y/o cultura" ocupa el segundo lugar de las respuestas (con un $28,3 \%$ ), en tanto que la "falta y no aplicación de leyes" es la primera causa (con un $37,1 \%$ de las respuestas), de acuerdo con los(as) entrevistados(as).

Consumo de energía. Se estima que en 2007 El Salvador alcanzó un consumo final de energía de 23.961 Mbep, 55,3 $\%$ cubierto principalmente por derivados del petróleo; 23 $\%$ por biomasa; $13,6 \%$ por electricidad, y $8,1 \%$ por otros (Cepal, 2007). En el 2008, los sectores de mayor consumo energético son el residencial, con $33 \%$ (la leña representó $67 \%)$; y el transporte, con $33 \%$, principalmente de derivados líquidos del petróleo; seguido por la industria con un $28 \%$, principalmente de derivados líquidos del petróleo (Cepal, 2009a).

\section{Estado ambiental - ¿Qué le está sucediendo al medio ambiente?}

La lógica del marco de referencia indica que las presiones antes detalladas producen un estado ambiental, en términos de contaminación atmosférica, erosión del suelo o deforestación. Como resultado, a continuación se presentan los aspectos que definen el estado ambiental.

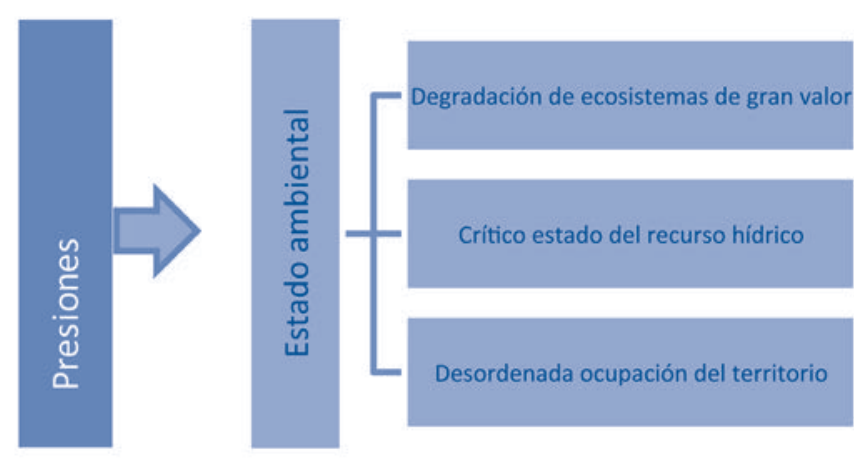

Figura 15. Presiones y estado ambiental

Degradación de ecosistemas de gran valor. La pérdida de cobertura en los cafetales a escala nacional, entre los años 1994 y 2010, fue de 41.028, con registros de 215.358 hectáreas en 1994 y 174.330 en 2010. Entre tanto, en la actualidad, el país apenas cuenta con un $27 \%$ de cobertura, que incluye un $13 \%$ de ecosistemas naturales, y cerca de un $9 \%$ de cafetales bajo sombra. Los bosques latifoliados deciduos y semideciduos, que representan un $8,5 \%$ en todo el territorio nacional, son los más afectados por la 
deforestación, con una pérdida de 40.471 ha, que comprende el $83,8 \%$ de toda la deforestación del país. En cambio, los bosques nebulosos y los manglares son los que menos pérdida han experimentado en este período (1998-2008) con 10 y 123 ha, respectivamente. Al respecto, el país pasó de tener unas 100.000 hectáreas de manglar en los años cincuenta a unas 40.000 en la actualidad: 38.534 poco intervenidas y unas 2.000 afectadas por azolvamiento o deforestación. Finalmente, solo el $4,72 \%$ de la superficie de El Salvador tiene algún estatus de área natural protegida, y el $9 \%$ de ecosistemas restantes no cuenta con ningún tipo de esquema de conservación o gestión sostenible (MARN, 2012b).

Crítico estado del recurso hídrico. Aunque la precipitación anual supone una importante oferta hídrica $\left(56.052,31 \mathrm{Mm}^{3}\right)$, la disponibilidad real de agua es baja y escasea a escala local debido a: la pérdida de la capacidad de regulación e infiltración del agua, el grave deterioro de la calidad del agua y la creciente variabilidad climática, que ha derivado en grandes cambios en la distribución espacial y temporal de las lluvias. Por otra parte, la disposición sanitaria de aguas residuales domésticas es un aspecto fundamental que se debe resolver, ya que tiene consecuencias importantes para la salud de la población. Solamente el $7 \%$ de las aguas residuales recibe algún tipo de tratamiento. Los resultados del estudio más reciente (MARN-DGOA, 2011) muestran que, de los 123 sitios evaluados, ninguno presenta calidad de agua "excelente", teniendo la mayoría de sitios agua de calidad "regular", desde el punto de vista de su calidad ambiental. De acuerdo con estos resultados, el MARN establece que la principal causa de contaminación del recurso hídrico en casi la mayoría de los sitios evaluados es la contaminación por aguas residuales domésticas sin tratamiento y la falta de saneamiento básico prevalentes en las zonas de aporte, lo cual se evidencia en la presencia de altas concentraciones de bacterias coliformes fecales en el agua, habiéndose encontrado valores de hasta 3.500.000 bacterias $/ 100 \mathrm{ml}$.

Desordenada ocupación del territorio. El uso inadecuado de las tierras, de acuerdo con su vocación natural, genera condiciones de bajo aprovechamiento y de deterioro de los suelos, lo cual conlleva a un conflicto de uso del suelo. Según datos del MARN, el $91 \%$ de las tierras del país se utilizan inapropiadamente; es decir, se usan sin atender la vocación del suelo, siendo que un $45 \%$ muestra un alto conflicto con el uso y un $45 \%$ muestra bajo conflicto. Apenas $9 \%$ de la tierra se utiliza de acuerdo con la vocación del suelo. Por otro lado, durante la formulación del Plan Nacional de Ordenamiento y Desarrollo Territorial (MOP, 2003), se estimó que un $40 \%$ del suelo salvadoreño presenta una erosionabilidad severa, 10 $\%$ una muy alta y $10 \%$ una alta.

\section{Impactos ambientales - ¿Cuáles son las consecuencias para el ambiente y la humanidad?}

Los principales impactos ambientales que genera el actual estado del ambiente (expresado en términos de la degradación de ecosistemas de gran valor, estado crítico del recurso hídrico y la desordenada ocupación del territorio) se enfocan sobre todo en la vulnerabilidad ambiental ante la variabilidad climática, y frente a las amenazas naturales.
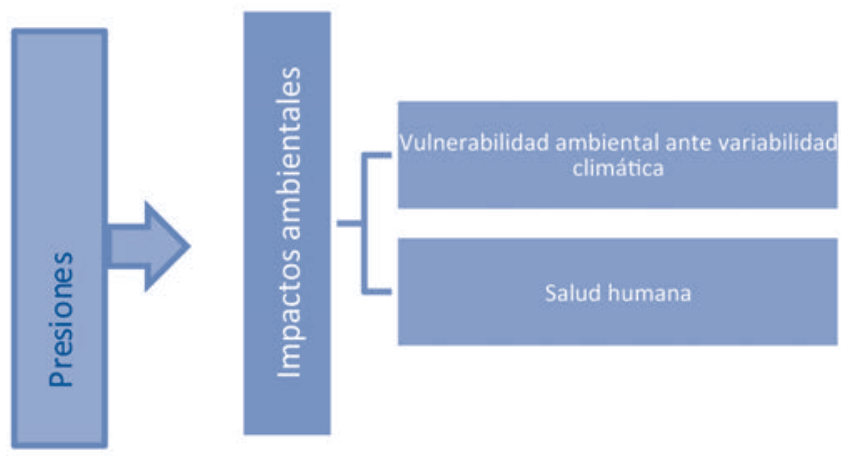

Figura 16. Estado ambiental e impactos

Asimismo, es importante destacar los efectos que genera la degradación ambiental, y específicamente el estado crítico del recurso hídrico sobre la salud humana. El 88,7 \% del territorio salvadoreño es susceptible a desastres naturales, y aproximadamente $95,4 \%$ de la población está en riesgo (MARN, 2011). De acuerdo con la Evaluación Mundial de Reducción de Desastres (UNISDR, 2009), El Salvador está clasificado como país "de alto riesgo" debido a las múltiples amenazas que enfrenta. El uso rural y urbano de la tierra ha incrementado la exposición y vulnerabilidad a desastres naturales.

Vulnerabilidad ambiental ante variabilidad climática. Del total de desastres naturales experimentados por el país durante los últimos años, los eventos climáticos han generado la mayor parte de los fallecimientos, más del $62 \%$; y casi la totalidad, entre 87 y $95 \%$ de los impactos totales. Solo en el período comprendido entre 1998 a 2010, 
El Salvador fue impactado por cinco eventos climáticos que provocaron un total de US\$2.333.1 millones en pérdidas. En 2011, con la depresión tropical E12, el país tuvo un total de US\$840 millones de pérdidas económicas, entre las que sobresalen US\$105 millones en agricultura. En ese mismo evento, se reportaron ocho puentes colapsados y 26 dañados; el $40 \%$ de las carreteras del país fueron dañadas; se registraron 947 escuelas dañadas; y se sufrieron 34 muertes humanas (MARN, 2012).

Salud humana. Según Romero (2002), una puntualización de los efectos económicos y sociales de la poca gestión ambiental que se hace de la red de aguas negras equivale a los daños económicos anuales por morbilidad y mortalidad por enfermedades relacionadas con el agua, que se estiman en US\$16.60 per cápita, y representan el 2,4\% de los ingresos por persona. Los costos en salud de un pobre abastecimiento de agua, saneamiento e higiene, por sí solos suman aproximadamente el $1 \%$ del PIB (Strukova, 2005). Las estimaciones demuestran que el $53 \%$ de los niños pobres en las áreas rurales sufren de enfermedades relacionadas con el agua entre uno y quince días del mes (especialmente de diarrea). En las áreas rurales, las familias sin conexión residencial de agua pasan entre 9 y $14 \%$ de su tiempo recolectando agua. REDI (2005) muestra que los pobres no son los principales beneficiarios de los esquemas de subsidios que intentan hacer del agua potable un rubro más accesible. Únicamente el $22 \%$ del costo anual de los subsidios de agua llegan a los hogares pobres.

\section{Respuestas - ¿Qué se está haciendo?}

El Salvador ha asumido la agenda ambiental internacional mediante la suscripción y ratificación de una serie de importantes convenciones, convenios y acuerdos internacionales y regionales. La Política Nacional del Medio Ambiente, aprobada en 2012, provee un marco general a las distintas políticas sectoriales ambientales bajo las siguientes líneas de acción: (1) restauración y conservación inclusiva de ecosistemas; (2) saneamiento ambiental integral; (3) gestión integrada del recurso hídrico; (4) incorporación de la dimensión ambiental en el ordenamiento territorial; (5) responsabilidad y cumplimiento ambiental; y (6) adaptación al cambio climático y reducción de riesgos. En julio del 2011, la Asamblea Legislativa ha aprobado la Ley de Ordenamiento y Desarrollo Territorial. Asimismo, se pueden detallar otras acciones de gobierno como respuesta a la degradación ambiental en áreas prioritarias como: (i) amenazas naturales y gestión de riesgos; (ii) energías alternativas; (iii) gestión de recursos hídricos; (iv) contaminación ambiental y manejo de desechos; (v) ecosistemas naturales; (vi) cambio climático; y (vii) gobernanza ambiental.

\section{Perspectivas para la sostenibilidad}

Como está demostrado, el sostenido crecimiento de la población, la expansión de la urbanización, la pobreza, los niveles de educación y el cambio de patrones de producción y consumo constituyen las fuerzas motrices constantes y evidentes que generan las principales presiones sobre el ambiente, a través del uso del suelo y de los recursos hídricos y la degradación de ecosistemas claves.

De seguir la tendencia actual, la demanda de bienes y servicios implicará un mayor deterioro ambiental, en especial en lo que respecta al uso de la tierra para fines agropecuarios, el consumo de agua y la emisión de contaminantes. El fomento del crecimiento económico del país demanda que se amplíe la infraestructura, en particular la vial y la energética, lo que se traduce en un respectivo costo de oportunidad de los bienes naturales.

Por lo tanto, los desafíos de los cambios rápidos en la tecnología, la globalización y las presiones ambientales crecientes requieren un marco estratégico para integrar el desarrollo sostenible al mercado, políticas y toma de decisiones. Se plantea, entonces, tomar en cuenta objetivos de sostenibilidad como: ecoeficiencia, tecnologías ambientales y toma de decisiones. Las primeras dos están orientadas a servir a los actores del desarrollo para que puedan ampliar sus horizontes y mejorar su participación en el mercado, tanto nacional como internacional. La toma de decisiones involucra la integración del desarrollo sostenible al proceso de toma de decisiones a escala nacional y el impacto de dichas decisiones en el contexto internacional. 


\section{OBJETIVOS DEL DESARROLLO SOSTENIBLE}

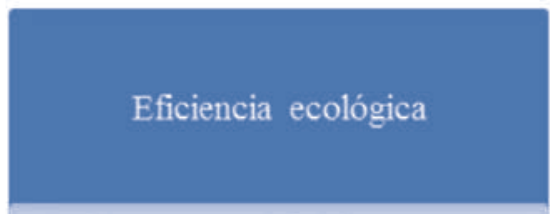

- Reforzar capacidad de sectores productivos.

- Implementar prácticas ecoeficientes.

\section{Tecnologias ambientales}

-Desarrollar tecnologías ambientales.

- Producir ventajas económicas.

- Generar beneficios ambientales.

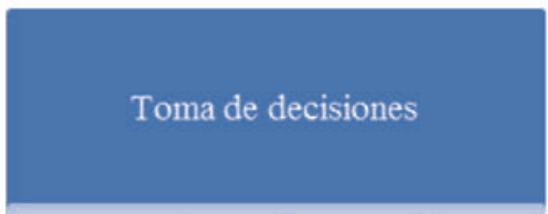

- Integrar desarrollo sostenible.

- Incluir políticas, planes y programas.

\section{INICIATIVAS}

Creando habilidades y capacidades de investigación y desarrollo.

Aplicando herramientas en zonas productivas.

Midiendo el éxito.
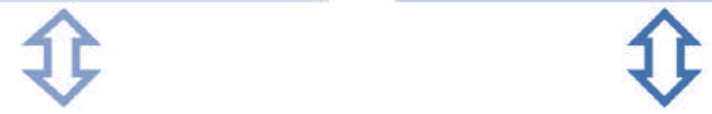

- Promoviendo innovación tecnológica.

- Trabajando en sociedad.

- Motivando nuevos alcances.
- Mejorando prácticas de planificación.

- Reforzando el desarrollo sostenible.

- Fortaleciendo la evaluación.

Figura 17. 
En definitiva, los tres objetivos arriba mencionados pueden ser alcanzados mediante iniciativas que estén dentro del alcance de cada uno de ellos, así:

\section{Tabla 2.}

Construyendo capacidades y habilidades de investigación y desarrollo.

Eficiencia ecológica

Tecnologías ambientales

Toma de decisiones
Aplicando las herramientas al mercado.

Midiendo el éxito.

Promoviendo innovación tecnológica.

Trabajando con sociedades.

Motivando nuevos enfoques.

Mejorando prácticas de planificación.

Mejorando el desarrollo sostenible.

Fortaleciendo evaluaciones.
Fortalecer las habilidades de investigación y el desarrollo en el área de la ecoeficiencia involucra la investigación aplicada, educación, tecnología y prácticas avanzadas. La aplicación de innovación y tecnologías probadas puede jugar un papel importante para que la industria sea más productiva.

Esto involucra la motivación de: la transferencia, adopción e implantación de herramientas de producción, prácticas, procesos y tecnologías ecoeficientes, en los niveles de producción y mercadeo para lograr una mejora en la productividad y el rendimiento ambiental.

La medida de rendimiento y benchmarks son importantes para evaluar la posición de competitividad. Ya que la ecoeficiencia es un tema innovador, es importante determinar si la implantación de iniciativas de este tipo realmente ha mejorado la productividad y el rendimiento. Para definir la sostentabilidad en términos de responsabilidades sociales corporativas, los factores económicos, sociales y ambientales son esenciales.

Promover la innovación es importante para cumplir las obligaciones ambientales y mejorar la productividad y la salud ambiental. Esto requeriría cerrar o reducir la brecha entre la innovación y la productividad.

Para asegurar que las tecnologías ambientales puedan responder de forma efectiva a las necesidades cambiantes del cliente objetivo, es esencial aumentar la conciencia entre sectores y actores. Esto sería construido sobre redes interinstitucionales e intergubernamentales, sectores industriales, socios y las ONG.

La complejidad de temas ambientales, tales como el del cambio climático, requieren enfoques innovadores para aplicar tecnologías ambientales. Solamente controlando las emisiones no se logrará una economía sostenible ambientalmente. El paradigma sobre comando y control deberá cambiarse a anticipación, evaluación y precaución.

La etapa de planificación del sistema administrativo involucra desarrollar las políticas, objetivos y metas para identificar y administrar las responsabilidades y obligaciones de las organizaciones. Esto también involucra el establecimiento de procedimientos, la designación de responsabilidades y la asignación de los recursos necesarios.

Las etapas de implantación y operación del sistema administrativo y toma de decisiones tratan de actuar sobre los compromisos asumidos durante la etapa de planificación. Esto incluye el desarrollo de procedimientos para cumplir con los compromisos y así lograr los objetivos de desarrollo sostenible.

El sistema administrativo involucra el desarrollo de metodología y procedimientos para evaluar el rendimiento organizacional, respecto a políticas, planes y programas. Esto incluye las lecciones aprendidas y las acciones correctivas tomadas para reducir la incidencia de errores. 


\section{Referencias}

PNUMA. 2007. Informe del Estado del Medio Ambiente de EI Salvador. GEO El Salvador 2003-2006. El Salvador.

Pintér, L.; Swanson, D.; Abdel-Jelil, I.; Nagatani, K.; Rahman, A.; Kok, M. 2007. Módulo de capacitación 5. Análisis integral de tendencias y políticas ambientales. Manual de capacitación para evaluación ambiental integral y elaboración de informes. PNUMA/IIDS. División de Evaluación y Alerta Temprana. P.O. Box 30552, Nairobi, 00100. Kenia.

PNUMA/ORLAC. 2008. Metodología para la elaboración de los informes GEO Ciudades. División de Evaluación y Alerta Temprana. Panamá.

Fondo de Población de las Naciones Unidas, UNFPA. 2010. El Salvador: transformaciones demográficas y sus implicaciones en las políticas públicas. Serie Cuadernos Salvadoreños de Población San Salvador, El Salvador.

Dirección General de Estadística y Censos, Digestyc. 2012. Encuesta de Hogares de Propósitos Múltiples 2011. Ciudad Delgado, San Salvador, El Salvador.

Minec y Digestyc (2009). Encuesta de Hogares de Propósitos Múltiples 2008. Base de datos. San Salvador: Ministerio de Economía, Dirección General de Estadística y Censos.

Sistema de las Naciones Unidas El Salvador. 2011. Evaluación común de país: El Salvador 2010. El Salvador.

MARN. 2011a. Mapa de los ecosistemas de El Salvador, actualización enero 2011. Proyecto de Consolidación y Administración de Áreas Protegidas (MARN-Pacap). El Salvador.
MARN. 2011b. Primera Encuesta Nacional sobre Conocimientos, Comportamientos, Percepciones y Prácticas de la población salvadoreña sobre Medio Ambiente y Riesgos. El Salvador.

MARN. 2012a. Política Nacional del Medio Ambiente. El Salvador.

MARN. 2012b. Propuesta de Estrategia de gestión de áreas naturales protegidas y corredores biológicos de El Salvador.

MARN/Dirección General del Observatorio Ambiental. 2011. Informe de calidad de agua de los ríos de El Salvador. Gerencia de Hidrología.

Administración Nacional de Acueductos y Alcantarillados, Anda. 2010. Boletín Estadístico. El Salvador.

Comisión Económica para América Latina y el Caribe, Cepal. 2007. Sistema de Información Económica Energética (SIEE). Olade.

Cepal. 2009a. La crisis de los precios del petróleo y su impacto en los países centroamericanos, México.

Ministerio de Obras Públicas (MOP) / Viceministerio de Vivienda y Desarrollo Urbano (VMVDU). 2003. Plan Nacional de Ordenamiento y Desarrollo Territorial. El Salvador.

United Nations International Strategy for Disaster Reduction Secretariat, UNISDR. 2009. Global Assessment Report on Disaster Risk Reduction. Geneva. http://www.preventionweb.net/english/hyogo/gar/report/index.php?id=9413

Strukova. 2005. The Cost of Environmental Degradation in El Salvador. Paper commissioned by World Bank. 